\title{
BMJ Open What is the most important determinant of cardiometabolic risk in 60-65-year- old subjects: physical activity-related behaviours, overall energy expenditure or occupational status? A cross-sectional study in three populations with different employment status in Poland
}

To cite: Soltysik BK, Kostka J, Karolczak K, et al. What is the most important determinant of cardiometabolic risk in 60-65-year-old subjects: physical activity-related behaviours, overall energy expenditure or occupational status? A cross-sectional study in three populations with different employment status in Poland. BMJ Open 2019;9:e025905. doi:10.1136/ bmjopen-2018-025905

Received 08 August 2018 Revised 04 July 2019 Accepted 09 July 2019

Check for updates

(C) Author(s) (or their employer(s)) 2019. Re-use permitted under CC BY-NC. No commercial re-use. See rights and permissions. Published by BMJ.

'Department of Geriatrics, Medical University of Łódź, Łódź, Poland

${ }^{2}$ Department of Physical Medicine, Medical University of Łódź, Łódź, Poland

${ }^{3}$ Department of Haemostatic Disorders, Medical University of Łódź, Łódź, Poland

Correspondence to Bartłomiej Konrad Sołtysik; bartlomiej.soltysik@umed. lodz.pl

\section{ABSTRACT:}

Objectives The aim of the study was to determine whether cardiovascular risk factors may differ according to occupational status and whether physical activity related to total energy expenditure (PA-EE) and related to healthrelated behaviours (PA-HRB) is associated with common cardiovascular risk factors or metabolic syndrome in preelderly subjects

Methods Three hundred subjects aged 60-65 were recruited and divided into three equal groups of whitecollar, blue-collar workers and unemployed subjects; $50 \%$ were women. The subjects were tested for major cardiovascular risk factors such as smoking, anthropometric indices, blood pressure, lipid levels, glucose, uric acid and homocysteine. PA-EE and PA-HRB were assessed with PA questionnaires.

Results Blue-collar workers displayed higher anthropometric indices, blood pressure and higher PAEE in comparison with other two groups. PA-HRB had a positive impact on body mass indices, lipids, glucose, uric acid and the prevalence of metabolic syndrome, with no such relationship observed for PA-EE.

Conclusions The greatest cardiovascular risk was observed in the blue-collar workers group. Only PA-HRB had a positive association with cardiometabolic risk profile. No relationship was observed for PA-EE. Thus, promoting everyday life and leisure time PA behaviours is crucial for preventing cardiometabolic risk in pre-elderly subjects, even in blue-collar workers with high work-related EE.

\section{INTRODUCTION}

Cardiometabolic diseases are a major cause of death and important reason of disability in developed countries. The occurrence of cardiovascular disease is related to the presence of risk factors. Smoking increases morbidity and mortality associated with cardiovascular diseases. ${ }^{12}$ Abdominal distribution
Strengths and limitations of this study

Three sex-matched and age-matched groups of subjects with different occupational status were recruited in the study.

- The paper promotes the idea that everyday life and leisure time physical activity (PA) behaviours are crucial for preventing cardiometabolic risk in pre-elderly subjects, even in blue-collar workers with high work-related energy expenditure.

- PA assessment tools (Seven Day Recall PA Questionnaire and the Stanford usual Activity Questionnaire) are self-reported subjective methods and the estimation of PA levels may be biased.

- The findings apply to balanced groups of CentralEuropean pre-elderly people according to employment status and these values may be different in other populations and cultures.

of fat has a negative impact on blood pressure (BP), lipid metabolism, glucose tolerance or insulin resistance. ${ }^{3}$ Obesity correlates with left ventricular hypertrophy, and weight reduction is a factor restoring normal heart muscle mass. ${ }^{4}$ Cardiovascular risk is related to high BP, especially to isolated systolic hypertension as a cause of coronary heart disease and stroke. ${ }^{5}{ }^{6}$ Hyperglycaemia is associated with increased risk of coronary heart disease. Diabetes worsens the long-term prognosis for life expectancy, ${ }^{7}$ as $2 / 3$ of diabetic deaths are related to cardiovascular disease. ${ }^{8}$ Atherosclerosis is positively correlated with low-density lipoprotein cholesterol (LDL-C) but negatively with high-density lipoprotein cholesterol (HDL-C) concentration. ${ }^{9}$ Homocysteine 
(Hcy) accelerates the development of atherosclerosis by enhancing the proliferation of vascular myocytes, endothelial dysfunction, oxidative stress and collagen synthesis, resulting in a deterioration of the elasticity of blood vessels. ${ }^{10}$

The elongation of the working period reinforces the need for research into how employment status influences the risk factors among seniors. The nature of work seems to have a direct impact on cardiovascular risk. Available data indicate that of various working groups, blue-collar workers demonstrate the greatest cardiovascular risk ${ }^{11}$ and that their workload may increase the risk of coronary heart disease. ${ }^{12}$ The prolonged working time of middle-aged men, especially those with current cardiovascular disease, can cause the progression of carotid atherosclerosis. ${ }^{13}$ There is some evidence that blue-collar work may be related to greater occurrence of increased BP than white-collar work, ${ }^{14}{ }^{15}$ even after adjustment for age, obesity or self-reported alcohol consumption. ${ }^{16}$ Furthermore, studies on some areas of work highlight the impact of job strain on arterial hypertension (HA). ${ }^{17}$ Psychosocial factors (low economic status, social isolation, chronic stress or depression) have a negative impact on cardiovascular risk, and significantly worsen the effects of treatment and prognosis of patients who already have developed the disease. ${ }^{18}$

Blue-collar workers have often been found to demonstrate a higher prevalence of daily smoking than whitecollar coworkers. ${ }^{19}{ }^{20}$ Some data indicate also that the unemployed are heavier tobacco users than professional workers. ${ }^{21}$ Subjects with occupations requiring lower qualifications may be more likely to demonstrate cardiovascular risk factors, such as obesity or lack of adequate PA. ${ }^{22}$ Prevention in psychological factors or restoring a healthy lifestyle can reduce the risk or moderate the progression of cardiovascular disease. ${ }^{23}$

Insufficient PA associated with a sedentary lifestyle increases cardiovascular risk, and an adequate level of activity can prolong the lifespan. ${ }^{24-26}$ Moderate or strong PA inhibits the development of atherosclerosis and reduces total mortality by $20 \%-25 \%,{ }^{27}$ which has been attributed to a range of effects such as reducing body weight, improving glucose tolerance ${ }^{28}$ or lipid profile. Blue-collar workers usually report high PA as a consequence of work conditions. ${ }^{29}$ The relationship observed between unemployment and low PA may be connected with higher susceptibility for cardiovascular diseases. ${ }^{30}$

It has recently been shown that subpopulations of older people differ with regard to their level of PA and its association with sociodemographic data and concomitant diseases. ${ }^{31}$ Furthermore, the relationship between PA and health profile may vary depending on the PA assessment methodology (PA-EE and PA-related health-related behaviours-PA-HRB). One of the most important questions in contemporary geriatric and occupational medicine is how socioeconomic status, workload and PA codetermine cardiovascular risk with regard to prolonged working time. Therefore, the aim of this work was to identify the occurrence of cardiovascular risk factors and diseases, and determine their relationships with different aspects of PA in three groups of pre-elderly: white-collar workers, blue-collar workers and unemployed seniors.

\section{METHODS \\ Subjects}

The study was conducted in three age-matched and sex-matched groups of inhabitants of the Łódź region aged 60-65 years. Participants were divided into three groups depending on the character of their professions: white-collar workers, blue-collar workers and unemployed subjects. The subjects were recruited through local media (TV, radio and newspapers). All the volunteers were initially checked for the basic recruitment criteria and classified according to the occupational status. As blue-collar workers were the least common group in this age range, they were qualified first for the study. An age-matched and sex-matched consecutive peer was assigned for each recruited worker from the whitecollar and unemployed groups. Each group included 100 volunteers (50 men and 50 women). The procedure for defining the type of profession has been described previously in a work concerning differences in the quality of work and life among the three senior groups. ${ }^{32}$

Subjects were asked about the presence of HA, diabetes mellitus type 2 (DM2), dyslipidaemia and smoking habit. BP was measured twice with an auscultation sphygmomanometer in accordance with the current guidelines. ${ }^{33}$ Fasting blood samples were drawn from the antecubital vein into test tubes. The blood serum was assayed spectrophotometrically for fasting glucose concentration, total cholesterol (TC), LDL-C, HDL-C, triglycerides (TG), uric acid (UA) (DIRUI CS 400, Changchun, China). Hcy was estimated in blood serum by immunochemiluminescence (Immulite 2000XPi analyser, Siemens, Germany). Blood morphology was evaluated with 5-Diff Sysmex XS-1000i haematological analyser (Sysmex, Kobe, Japan). Weight and height were measured in participants while barefoot, and waist and hip circumference were measured ${ }^{34}$; body mass index (BMI) and waist-hip ratio (WHR) were then calculated based on the results. Metabolic syndrome was assessed according to International Diabetes Federation. ${ }^{35}$

\section{PA assessment}

PA was assessed by two popular PA questionnaires: the Seven-Day Recall PA Questionnaire ${ }^{36}$ and the Stanford Usual Activity Questionnaire. ${ }^{37}$ Both questionnaires used in the present study have been previously described in detail. ${ }^{38}$ Both have demonstrated high validity in older individuals against doubly labelled water and have been assessed in the present study in accordance with standardised protocols. ${ }^{3139}$

All participants were informed that the questionnaires were anonymous and that only the researchers will have access to the results. For the Seven-Day Recall PA Questionnaire, a list of examples of different level of activities 
was included. Additionally, if in doubt, participants were allowed to ask questions to the interviewer.

The Seven-Day Recall PA Questionnaire determines the hours spent sleeping for the week, sums up the time spent in light (activities with EE of $1.5 \mathrm{kcal} . \mathrm{min}^{-1}$ ), moderate (activities with EE of $4 \mathrm{kcal} . \mathrm{min}^{-1}$ ), hard (activities with $\mathrm{EE}$ of $6 \mathrm{kcal}^{-1} \mathrm{~min}^{-1}$ ) and very hard (activities with $\mathrm{EE}$ of 10 kcal.min ${ }^{-1}$ ) activities and estimates overall weekly EE through analysis of PA during the previous 7 days. The Seven-Day Recall Total score (total EE over past week$\mathrm{kcal} \cdot \mathrm{kg}^{-1} \cdot \mathrm{day}^{-1}$ ) was then calculated and used for further comparisons as PA-EE.

The Stanford moderate index allows an assessment of health-related PA behaviours of light and moderate intensity. The respondents indicate the type of behaviour typical of their exercise habits: climbing the stairs instead of using the elevator, walking instead of driving for a short distance, parking the car further away from the destination in order to approach on foot, walking before or after lunch or dinner, exiting the bus or tram a stop earlier in order to walk the remaining distance, or performing other activities of a similar nature. In the Stanford Hard (vigorous) index, the respondent indicates the following activities performed regularly for at least the last 3 months: jogging or running at least 10 miles per week, play strenuous racquet sports at least 5 hours per week, play other strenuous sports at least 5 hours per week, ride a bicycle at least 50 miles per week, swim at least 2 miles per week. The Stanford Moderate (six habitual moderate activities; scoring points $0-6$ ) and Hard (five habitual intensive activities; scoring points $0-5$ ) indices were calculated as a numerical sum of points for each activity and used for further comparisons. These two PA indices are expressed as PA-HRB I and PA-HRB II, respectively.

\section{Statistical analysis}

Data were verified for normality of distribution and equality of variances. The one-way analysis of variance with Tukey posthoc testing, Kruskal-Wallis test and $3 \times 2$ $\chi^{2}$ test were used to compare the three groups. Student's t-test, Mann-Whitney test and $\chi^{2}$ test $(2 \times 2$ with Yates' correction) were used to make comparisons between the two groups with applied Bonferroni correction. The correlations were assessed with Spearman's correlation coefficients. The results of the quantitative variables are presented as mean $\pm \mathrm{SD}$ or median (IQR). Multivariate analyses were performed to identify factors independently predicting cardiometabolic risk, general linear models were constructed for the main cardiovascular risk factors and logistic regression was employed for dichotomised variables. All the variables statistically significant in bivariate relationships were entered into the multivariate analyses. Age, sex, occupational status, PA variables, smoking and drugs were taken into consideration as potential independent variables. Non-normally distributed variables were log-transformed for the purpose of multivariate analyses; however, the results are presented in standard values. PA-HRB II was dichotomised for multivariate analyses as no PA-HRB II versus at least one PA-HRB II. The limit of significance was regarded as $\mathrm{p} \leq 0.05$ for all analyses. Statistical analysis were performed using Statistica V.12 software.

\section{Patient and public involvement statement}

The study design was based on the fact that Łódź region is one with the fastest ageing population in Europe. The study was important for the understanding of the impact of occupational status on cardiovascular risk among pre-elderly seniors. The respondents obtained information on individual test results. In addition, they are informed about the results obtained on the basis of the collected data.

No additional involvement of patients has been stated.

\section{RESULTS}

Table 1 presents differences between the three working groups. The median age of the unemployed subjects was slightly higher than those of both working groups. Whitecollar workers were better $(\mathrm{p}<0.001)$ educated than bluecollar workers and the unemployed subjects. Blue-collar workers presented statistically significantly higher WHR, systolic blood pressure (SBP), diastolic blood pressure (DBP) and PA-EE than the other two groups. Blue-collar workers also presented a significantly higher platelet count in comparison with white-collar workers $(\mathrm{p}=0.02)$.

The prevalence of cardiovascular diseases and risk factors throughout the whole studied population was as follows: hypercholesterolemia: 66\%, HA: $52.33 \%$, DM2: $11.67 \%$, previous myocardial infarction (MI): $5 \%$, ischaemic heart disease (IHD): $14.33 \%$, previous stroke: $4 \%$, obesity (counted as a BMI equal or greater than $30 \mathrm{~kg}^{*} \mathrm{~m}^{-}$ $\left.{ }^{2}\right)$ : $31.67 \%$, and metabolic syndrome: $65 \%$ of subjects. No differences were seen between the three groups regarding the incidence of diseases, smoking prevalence or drug intake.

Regarding the female respondents, the unemployed subjects were significantly older than working subjects, and those in white-collar work were statistically significantly better educated than the other two groups. Bluecollar workers were statistically significantly more obese (WHR), with higher SBP and PA-EE values, than the white-collar and unemployed groups. The women in bluecollar work had statistically significantly higher platelet count and DBP than those in white-collar positions, and displayed significantly higher UA concentration than unemployed respondents. Table 2A displays differences between groups of women in more detail.

Regarding the men, white-collar workers were statistically significantly better educated than blue-collar workers and unemployed men. Blue-collar workers had statistically significantly higher SBP, DBP and PA-EE than the other two groups. Unemployed men had higher TG $(\mathrm{p}=0.01)$ and lower HDL-C $(\mathrm{p}=0.03)$ than the white-collar workers. These differences are highlighted in table $2 \mathrm{~b}$. No differences were found between the three groups 
Table 1 Comparison of the major anthropometric variables, cardiovascular risk factors, diseases and drug intake between the three groups

\begin{tabular}{|c|c|c|c|}
\hline Variable & $\begin{array}{l}\text { White-collar workers } \\
\text { Mean } \pm S D \text { or median } \\
\text { (quartiles) } n=100\end{array}$ & $\begin{array}{l}\text { Blue-collar workers } \\
\text { Mean } \pm S D \text { or median } \\
\text { (quartiles) } n=100\end{array}$ & $\begin{array}{l}\text { Unemployed subjects } \\
\text { Mean } \pm \text { SD or median } \\
\text { (quartiles) } n=100\end{array}$ \\
\hline Age (years) & $62.5(61-64)$ & $62(61-64)$ & $63(63-64)^{*}(p<0.001)+(p<0.001)$ \\
\hline Education (years) & $15.5(12.5-17)$ & $12(11-13)^{*(p<0.001)}$ & $12(11-14.5)^{*}{ }^{*}(p<0.001)$ \\
\hline $\mathrm{BMI}\left(\mathrm{kg} / \mathrm{m}^{2}\right)$ & $27.7 \pm 3.9$ & $28.2(25.5-31.5)$ & $27.5(23.9-30.8)$ \\
\hline Waist circumference (cm) & $95(87.5-102)$ & $97(90-107.7)^{*(p=0.03)}$ & $96(83-105)$ \\
\hline WHR & $0.9(0.8-1.0)$ & $0.9 \pm 0.1^{*(p=0.01)}$ & $0.9(0.8-1.0)^{\dagger(p=0.01)}$ \\
\hline TC (mg/dL) & 207.4 (178.6-233.5) & 203.5 (172.5-235.0) & $203.1(167.1-239.3)$ \\
\hline LDL (mg/dL) & $126.5(103.3-152.1)$ & $130.9 \pm 38.6$ & $127.4 \pm 38.8$ \\
\hline $\mathrm{HDL}(\mathrm{mg} / \mathrm{dL})$ & $50.0(42.5-57.6)$ & $46.9(40.7-56.2)$ & $47.9(39.9-59.6)$ \\
\hline $\mathrm{TG}(\mathrm{mg} / \mathrm{dL})$ & 107.7 (74.9-148.9) & $109.2(74.5-158.7)$ & 116.5 (82.2-173.9) \\
\hline Glucose (mg/dL) & $97.2(90.1-110.3)$ & $101.9(93.7-113.2)$ & $98.8(90.8-111.5)$ \\
\hline Uric acid (mg/dL) & $4.9 \pm 1.2$ & $4.8(4.0-5.7)$ & $4.8 \pm 1.4$ \\
\hline Homocysteine $(\mu \mathrm{mol} / \mathrm{L})$ & $14.0(12.5-17.0)$ & $14.45(12.2-16.8)$ & $14.8(12.9-17.1)$ \\
\hline Blood platelets $\left(10^{3} / \mathrm{mm}^{3}\right)$ & $207.0 \pm 48.1$ & $223.6 \pm 50.1^{*(p=0.02)}$ & $214.5(173.0-250.5)$ \\
\hline SBP $(\mathrm{mm} \mathrm{Hg})$ & $133.5 \pm 17.0$ & $143.4 \pm 17.0^{*}(\mathrm{p}<0.001)$ & $134.5 \pm 16.0^{\dagger(p<0.001)}$ \\
\hline $\mathrm{DBP}(\mathrm{mm} \mathrm{Hg})$ & $80.0(72.0-88.0)$ & $87.0 \pm 11.7^{*(p<0.001)}$ & $81.9 \pm 12.4^{+(p=0.001)}$ \\
\hline Metabolic syndrome & $63 \%$ & $69 \%$ & $63 \%$ \\
\hline PA-EE (kcal/kg/day) & $41.3(37.3-48.6)$ & $49.6 \pm 7.6^{*(p<0.001)}$ & $41.0(37.5-44.7)^{t(p<0.001)}$ \\
\hline Stanford moderate PA-HRB I & $3.0(1.0-4.0)$ & $3.0(1.0-4.0)$ & $3.0(2.0-4.0)$ \\
\hline Stanford hard PA-HRB II & $0.0(0.0-0.0)$ & $0.0(0.0-0.0)$ & $0.0(0.0-0.0)$ \\
\hline $\begin{array}{l}\text { Stanford hard PA-HRB II (at } \\
\text { least one) }\end{array}$ & $17 \%$ & $12 \%$ & $18 \%$ \\
\hline Diseases and drugs & $\mathbf{n}$ & $\mathbf{n}$ & n \\
\hline $\mathrm{HA}$ & 50 & 53 & 54 \\
\hline Hypercholesterolemia & 60 & 67 & 71 \\
\hline Smoking & 20 & 26 & 23 \\
\hline DM2 & 10 & 13 & 12 \\
\hline MI & 5 & 4 & 6 \\
\hline IHD & 11 & 13 & 19 \\
\hline Stroke & 4 & 4 & 4 \\
\hline Obesity & 30 & 33 & 32 \\
\hline Antiplatelet drugs & 16 & 15 & 23 \\
\hline B-blocker & 26 & 28 & 31 \\
\hline Ca-blocker & 13 & 13 & 7 \\
\hline $\begin{array}{l}\text { Angiotensin converting enzyme } \\
\text { inhibitor }\end{array}$ & 21 & 27 & 22 \\
\hline Angiotensin II receptor blocker & 12 & 6 & 9 \\
\hline Diuretics & 24 & 23 & 10 \\
\hline Hypolipemic drugs & 21 & 26 & 30 \\
\hline Antidiabetics & 9 & 14 & 11 \\
\hline
\end{tabular}

*Statistically different from white-collar.

†Statistically different from blue-collar workers.

BMI, body mass index; DBP, diastolic blood pressure; DM2, diabetes mellitus type 2; HA, arterial hypertension; HDL-C, high-density lipoprotein cholesterol; IHD, ischaemic heart disease; LDL-C, low-density lipoprotein cholesterol; MI, myocardial infarction; PA-EE, physical activity related to energyexpenditure; PA-HRB I, physical activity moderate health related behaviours; PA-HRB II, physical activity hard health related behaviours; SBP, systolic blood pressure; TC, total cholesterol; TG, triglyceride; WHR, waist-hip ratio. 
Table 2A Comparison of the major anthropometric variables, cardiovascular risk factors, diseases and drug intake between the three groups of women

\begin{tabular}{|c|c|c|c|}
\hline Variable & $\begin{array}{l}\text { White-collar female } \\
\text { workers } \\
\text { Mean } \pm S D \text { or median } \\
\text { (quartiles) } n=50\end{array}$ & $\begin{array}{l}\text { Blue-collar female } \\
\text { workers } \\
\text { Mean } \pm S D \text { or median } \\
\text { (quartiles) } n=50\end{array}$ & $\begin{array}{l}\text { Unemployed females } \\
\text { Mean } \pm \text { SD or median (quartiles) } \\
\mathrm{n}=50\end{array}$ \\
\hline Age (years) & $62(61-64)$ & $62(61-63)$ & $63(63-64)^{*}{ }^{*}(p<0.001)+(p<0.001)$ \\
\hline Education (years) & $14.5(12-17)$ & $13(11-14)^{*(p<0.001)}$ & $13(12-16)^{*}{ }^{*}(p=0.04)$ \\
\hline BMI (kg/m²) & $27.6 \pm 4.4$ & $28.24(25.3-31.3)$ & $27.0(23.4-31.6)$ \\
\hline Waist circumference (cm) & $88.5(81-97)$ & $93(86-100)$ & $85.5(77.5-100)$ \\
\hline WHR & $0.8 \pm 0.1$ & $0.9 \pm 0.1^{*(p=0.01)}$ & $0.8 \pm 0.1^{\dagger(p<0.001)}$ \\
\hline $\mathrm{TC}(\mathrm{mg} / \mathrm{dL})$ & 214.5 (185.0-249.0) & $221.3 \pm 51.5$ & $216.4 \pm 44.3$ \\
\hline $\mathrm{LDL}(\mathrm{mg} / \mathrm{dL})$ & $140.4(104.9-156.3)$ & $143.7 \pm 41.1$ & $134.5 \pm 41.9$ \\
\hline $\mathrm{HDL}(\mathrm{mg} / \mathrm{dL})$ & $52.9(45.4-69.0)$ & $51.2(45.2-51.9)$ & $57.0 \pm 14.6$ \\
\hline $\mathrm{TG}(\mathrm{mg} / \mathrm{dL})$ & $107.9(78.6-159.8)$ & $114.7(74.0-158.7)$ & $113.0(76.8-159.4)$ \\
\hline Glucose (mg/dL) & $95.8(89.0-105.6)$ & $101.6(92.6-108.9)$ & $94.7(88.7-106.9)$ \\
\hline Uric acid (mg/dL) & $4.4 \pm 1.2$ & $4.3(3.9-5.1)$ & $3.8(3.2-4.9)^{t(p=0.01)}$ \\
\hline Homocysteine ( $\mu \mathrm{mol} / \mathrm{L})$ & $13.3(11.9-16.2)$ & $14.25(12.1-15.9)$ & $14.3(12.7-16.0)$ \\
\hline Blood platelets $\left(10^{3} / \mathrm{mm}^{3}\right)$ & $220.1 \pm 45.9$ & $246.2 \pm 46.7^{*(p=0.005)}$ & $224.5(196-256)$ \\
\hline $\mathrm{SBP}(\mathrm{mm} \mathrm{Hg})$ & $130.5 \pm 15.48$ & $140.5 \pm 15.1^{*(p=0.003)}$ & $133.1 \pm 13.2^{\dagger(p=0.01)}$ \\
\hline $\mathrm{DBP}(\mathrm{mm} \mathrm{Hg})$ & $78.2 \pm 11.6$ & $84.6 \pm 9.9^{*}(p=0.004)$ & $80.5 \pm 11.9$ \\
\hline Metabolic syndrome & 27 (54\%) & $30(60 \%)$ & 22 (44\%) \\
\hline PA-EE (kcal/kg/ day) & $44.1(38.7-48.7)$ & $50.52 \pm 7.33^{*(p<0.001)}$ & $41.0(38.1-43.8)^{\dagger(p<0.001)}$ \\
\hline Stanford moderate PA-HRB I & $3.0(2.0-4.0)$ & $3.0(2.0-4.0)$ & $3.0(2.0-4.0)$ \\
\hline Stanford hard PA-HRB II & $0.0(0.0-0.0)$ & $0.0(0.0-0.0)$ & $0.0(0.0-0.0)$ \\
\hline $\begin{array}{l}\text { Stanford hard PA-HRB II (at least } \\
\text { one) }\end{array}$ & $8(16 \%)$ & $5(10 \%)$ & $10(20 \%)$ \\
\hline Diseases and drugs & n & n & $\mathbf{n}$ \\
\hline $\mathrm{HA}$ & 24 & 23 & 21 \\
\hline Hypercholesterolemia & 34 & 35 & 36 \\
\hline Smoking & 13 & 12 & 6 \\
\hline DM2 & 4 & 7 & 5 \\
\hline $\mathrm{Ml}$ & 1 & 0 & 1 \\
\hline IHD & 6 & 5 & 6 \\
\hline Stroke & 3 & 2 & 1 \\
\hline Obesity & 17 & 16 & 14 \\
\hline Antiplatelet drugs & 6 & 9 & 13 \\
\hline B-blocker & 15 & 14 & 15 \\
\hline Ca-blocker & 5 & 5 & 2 \\
\hline $\begin{array}{l}\text { Angiotensin converting enzyme } \\
\text { inhibitor }\end{array}$ & 10 & 13 & 10 \\
\hline Angiotensin II receptor blocker & 10 & 3 & 4 \\
\hline Diuretics & 12 & 12 & 5 \\
\hline Hypolipemic drugs & 9 & 12 & 13 \\
\hline Antidiabetics & 3 & 7 & 5 \\
\hline
\end{tabular}

Continued 
Table 2A Continued

\section{Variable}

White-collar female workers

Mean \pm SD or median (quartiles) $\mathrm{n}=50$
Blue-collar female workers

Mean \pm SD or median $\quad$ Mean \pm SD or median (quartiles) (quartiles) $\mathrm{n}=50$

\section{$\mathrm{n}=50$}

*Statistically different from white-collar.

†Statistically different from blue-collar workers.

.BMI, body mass index;DBP, diastolic blood pressure; DM2, diabetes mellitus type 2; HA, arterial hypertension; HDL-C, high-density lipoprotein cholesterol; IHD, ischaemic heart disease; LDL-C, low-density lipoprotein cholesterol; PA-EE, physical activity related to energy expenditure; PA-HRB I, physical activity moderate health-related behaviours; PA-HRB II, physical activity hard health-related behaviours; SBP, systolic blood pressure; TC, total cholesterol; TG, triglyceride; WHR, waist-hip ratio.

Table 2B Comparison of the major anthropometric variables, cardiovascular risk factors, diseases and drug intake between the three groups of men

\begin{tabular}{|c|c|c|c|}
\hline Variable & $\begin{array}{l}\text { White-collar male } \\
\text { workers } \\
\text { Mean } \pm S D \text { or median } \\
\text { (quartiles) } n=50\end{array}$ & $\begin{array}{l}\text { Blue-collar male workers } \\
\text { Mean } \pm S D \text { or median } \\
\text { (quartiles) } n=50\end{array}$ & $\begin{array}{l}\text { Unemployed men } \\
\text { Mean } \pm S D \text { or median (quartiles) } \\
n=50\end{array}$ \\
\hline Age (years) & $63(61-64)$ & $63(61-65)$ & $63(63-65)$ \\
\hline Education (years) & $16(13-17)$ & $12.0(11.0-13.0)^{*}(p<0.001)$ & $12.0(11.0-13.0)^{*}(p<0.001)$ \\
\hline $\mathrm{BMI}\left(\mathrm{kg} / \mathrm{m}^{2}\right)$ & $27.8 \pm 3.5$ & $28.0(25.5-32.1)$ & $27.7 \pm 4.3$ \\
\hline Waist circumference $(\mathrm{cm})$ & $98.5(94-106)$ & $103(95-114.5)$ & $102(94-109.5)$ \\
\hline WHR & $1.0 \pm 0.1$ & $1.0 \pm 0.1$ & $1.0 \pm 0.1$ \\
\hline TC (mg/dL) & $197.8 \pm 35.8$ & $190.3 \pm 37.4$ & $192.5 \pm 40.1$ \\
\hline LDL (mg/dL) & $124.9 \pm 30.8$ & $117.9 \pm 31.3$ & $120.1 \pm 34.1$ \\
\hline $\mathrm{HDL}(\mathrm{mg} / \mathrm{dL})$ & $48.2(39.3-54.0)$ & $44.7(39.2-52.5)$ & $41.5(36.6-48.4)^{*}{ }^{*}(p=0.03)$ \\
\hline $\mathrm{TG}(\mathrm{mg} / \mathrm{dL})$ & $104.4(70.9-139.3)$ & $106.0(74.9-158.6)$ & $123.1(88.3-188.5)^{*}(p=0.01)$ \\
\hline Glucose (mg/dL) & $99.2(92.2-112.4)$ & $102.3(96.1-114.7)$ & $102.3(93.9-114.1)$ \\
\hline Uric acid (mg/dL) & $5.3 \pm 1.0$ & $5.3 \pm 1.2$ & $5.5 \pm 1.1$ \\
\hline Homocysteine ( $\mu \mathrm{mol} / \mathrm{L})$ & $16.0 \pm 3.7$ & $14.7(12.7-17.5)$ & $15.9 \pm 3.9$ \\
\hline Blood platelets $\left(10^{3} / \mathrm{mm}^{3}\right)$ & $193.7 \pm 47.0$ & $201.5 \pm 43.3$ & $187.5(165.0-237.0)$ \\
\hline $\mathrm{SBP}(\mathrm{mm} \mathrm{Hg})$ & $135(125-145)$ & $146.3 \pm 18.4^{*(p=0.002)}$ & $135.9 \pm 18.3^{\dagger(p=0.008)}$ \\
\hline $\mathrm{DBP}(\mathrm{mm} \mathrm{Hg})$ & $82(75-93)$ & $89.4 \pm 12.9^{*}(p=0.03)$ & $83.2 \pm 12.8^{t(p=0.01)}$ \\
\hline Metabolic syndrome & $36(72 \%)$ & $39(78 \%)$ & $41(82 \%)$ \\
\hline PA-EE (kcal/kg/ day) & $40.0(36.6-48.1)$ & $48.7 \pm 7.9^{*(p<0.001)}$ & $40.9(37.3-45.0))^{\dagger(p<0.001)}$ \\
\hline Stanford moderate PA-HRB I & $3.0(1.0-4.0)$ & $2.5(1.0-4.0)$ & $2.5(1.0-4.0)$ \\
\hline Stanford hard PA-HRB II & $0.0(0.0-0.0)$ & $0.0(0.0-0.0)$ & $0.0(0.0-0.0)$ \\
\hline $\begin{array}{l}\text { Stanford hard PA-HRB II (at least } \\
\text { one) }\end{array}$ & $9(18 \%)$ & $7(14 \%)$ & $8(16 \%)$ \\
\hline Diseases and drugs & $\mathbf{n}$ & $\mathbf{n}$ & $\mathbf{n}$ \\
\hline $\mathrm{HA}$ & 26 & 30 & 33 \\
\hline Hypercholesterolemia & 26 & 32 & 35 \\
\hline Smoking & 7 & 14 & 17 \\
\hline DM2 & 6 & 6 & 7 \\
\hline Ml & 4 & 4 & 5 \\
\hline IHD & 5 & 8 & 13 \\
\hline Stroke & 1 & 2 & 3 \\
\hline Obesity & 13 & 17 & 18 \\
\hline Antiplatelet drugs & 10 & 6 & 10 \\
\hline B-blocker & 11 & 14 & 16 \\
\hline Ca-blocker & 8 & 8 & 5 \\
\hline
\end{tabular}

Continued 


\begin{tabular}{|c|c|c|c|}
\hline Variable & $\begin{array}{l}\text { White-collar male } \\
\text { workers } \\
\text { Mean } \pm \text { SD or median } \\
\text { (quartiles) } n=50\end{array}$ & $\begin{array}{l}\text { Blue-collar male workers } \\
\text { Mean } \pm S D \text { or median } \\
\text { (quartiles) } n=50\end{array}$ & $\begin{array}{l}\text { Unemployed men } \\
\text { Mean } \pm \text { SD or median (quartiles) } \\
n=50\end{array}$ \\
\hline $\begin{array}{l}\text { Angiotensin converting enzyme } \\
\text { inhibitor }\end{array}$ & 11 & 14 & 12 \\
\hline Angiotensin II receptor blocker & 2 & 3 & 5 \\
\hline Hypolipemic drugs & 12 & 14 & 17 \\
\hline Antidiabetics & 6 & 7 & 6 \\
\hline
\end{tabular}

*Statistically different from white-collar. †Statistically different from blue-collar workers.

.BMI, body mass index; DBP, diastolic blood pressure; DM2, diabetes mellitus type 2; HA, arterial hypertension; HDL-C, high-density lipoprotein cholesterol; PA-HRB I, physical activity moderate health-related behaviours; IHD, ischaemic heart disease; PA-HRB II, physical activity hard health-related behaviours; LDL-C, low-density lipoprotein cholesterol; PA-EE, physical activity related to energy expenditure; SBP, systolic blood pressure; TC, total cholesterol; TG, triglyceride; WHR, waist-hip ratio.

concerning PA-HRB, incidence of diseases, smoking prevalence or drug intake either with or without division by sex.

Table 3 describes correlations between PA-EE, PA-HRB and major cardiovascular risk factors. In the whole studied group, PA-EE was directly related to SBP. Subjects with higher PA-HRB I showed lower body mass indices, lower TG, glucose, UA concentration and had a lower incidence of metabolic syndrome. Higher PA-HRB II was related to lower body mass indices, lower TG, glucose, higher HDL-C and lower frequency of metabolic syndrome. Women with higher PA-EE had higher WHR, while those with higher PA-HRB (both moderate and hard) had lower body mass indices (BMI and BMI/ WHR, respectively). Higher PA-HRB I correlated with lower TG, glucose and UA; higher PA-HRB II was associated with higher HDL-C and lower glucose and UA concentration. In men, higher PA-EE was related to lower concentration of Hcy and higher SBP. Both PA-HRB I and PA-HRB II correlated negatively with WHR, and PA-HRB II also correlated negatively with BMI. Higher PA-HRB II was associated with lower TG, higher HDL-C and lower frequency of metabolic syndrome (table 3).

Interestingly, in the whole group, PA-HRB I and PA-HRB II levels correlated with PA-EE $(\mathrm{r}=0.17 ; \mathrm{p}=0.003$ and $\mathrm{r}=0.18 ; \mathrm{p}=0.002$, respectively) but not with each other. PA-EE was related to PA-HRB I and PA-HRB II in men but only to PA-HRB II in women (not shown in the table).

\section{Multivariate analyses}

General linear model and logistic regression were further used to select variables that independently predict major cardiovascular risk factors (table 4). PA-HRB I was an independent predictor of lower BMI, WHR, glucose, UA and frequency of metabolic syndrome. PA-HRB II decreased BMI, WHR, TG, glucose and frequency of metabolic syndrome while increasing HDL-C level. Whitecollar workers had lower SBP and DBP while blue-collar workers had higher SPB, DBP as well as WHR when compared with unemployed subjects. Women had higher TC, HDL-C, LDL-C and Hcy but lower WHR, glucose, UA, SBP, DBP and frequency of metabolic syndrome as compared with men. Smoking was an independent predictor of higher TG levels. Hypolipemic drugs use was related to lower TC and LDL-C, angiotensin converting enzyme inhibitors use to lower LDL-C and antidiabetics intake was associated with lower Hcy levels (table 4). In multivariate models PA-EE had no impact on presented cardiovascular risk factors.

\section{DISCUSSION}

The major finding of the present study is that in 60-65 year-old subjects, PA-related behaviours have a greater influence on cardiometabolic risk than overall EE or employment status. The prevalence of cardiometabolic diseases is similar across this group of pre-elderly subjects, independent of workload, with no apparent relationship being found between PA and health status. However, the prevalence of cardiometabolic risk factors was found to vary depending on workload, with the group of bluecollar workers displaying the worst profile.

Blue-collar workers presented higher indices for body mass than white-collar workers and unemployed subjects, with WHR being significantly greater. Nevertheless, all three groups demonstrated similar levels of obesity, defined as BMI equal or greater than $30 \mathrm{~kg} / \mathrm{m}^{2}$. Some indications exist that occupational PA may have a protective impact against obesity; ${ }^{340}$ however, our data indicate that higher body mass indices and higher PA-EE scores were observed in the blue-collar group, which is coherent with some literature data. ${ }^{30}$

As the highest values of $\mathrm{BP}$ were observed in the bluecollar group, hypertension may be associated with physical workload. ${ }^{41}$ Despite demonstrating a similar ratio 


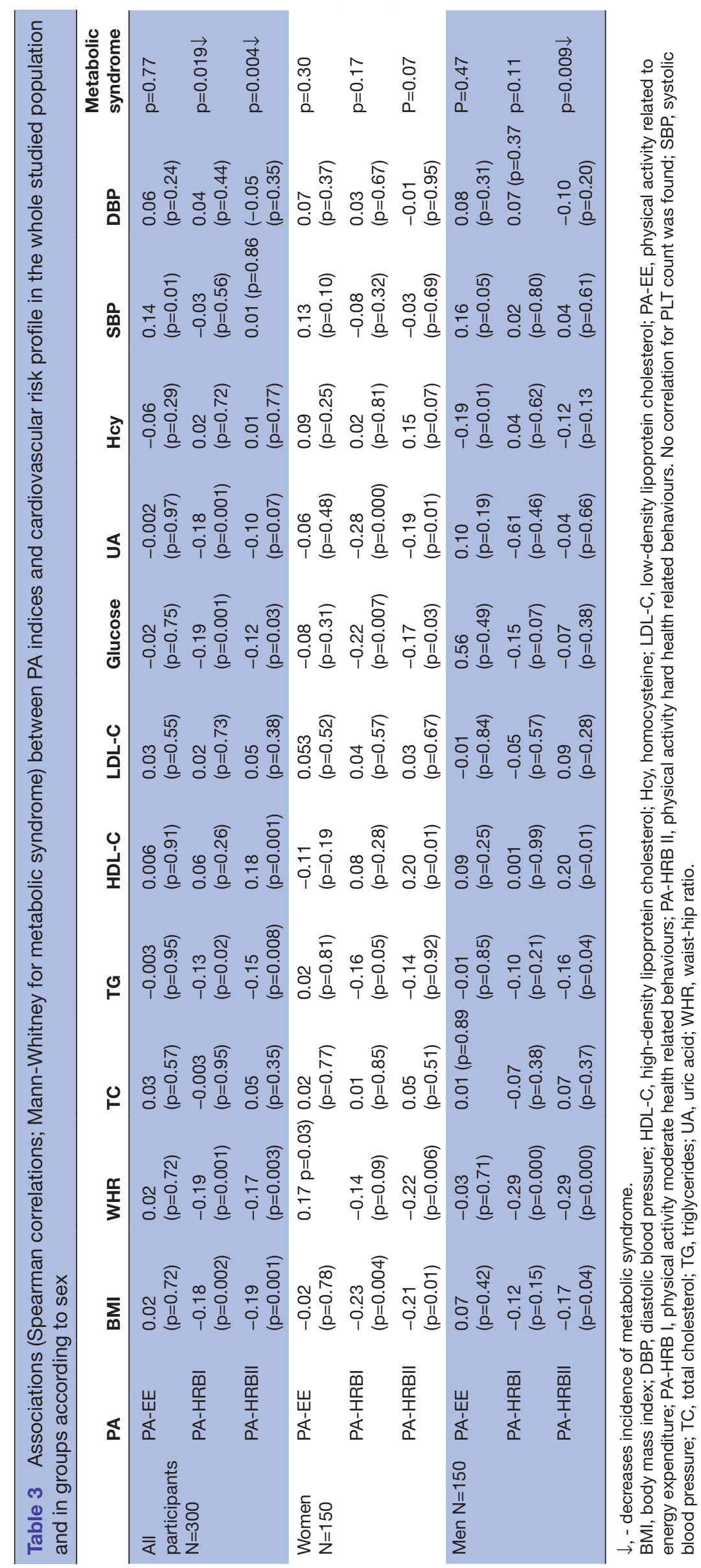




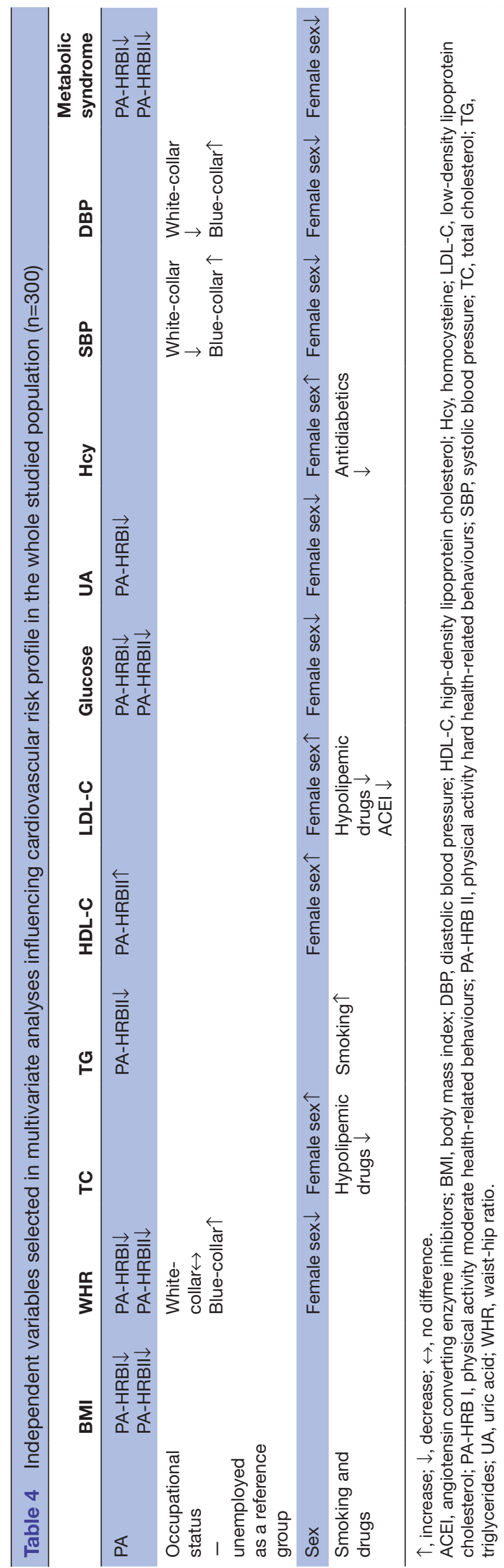

of hypertensive treatment and diagnosed HA to the other groups, blue-collar workers presented higher BP. Engaging in physical work each working day for several hours may result in elevated BP and increased cardiovascular risk. ${ }^{142}$ Furthermore, some studies characterise blue-collar workers with the highest rate of obesity, another important risk factor for hypertension. ${ }^{43}$ Importantly, despite the fact that over $50 \%$ of subjects were diagnosed with HA, BP measures indicate that bluecollar workers may be still underdiagnosed and undertreated. Furthermore, blue-collar work may be associated with other sex-related cardiovascular risk factors, that is, higher glucose and UA concentration among women and greater lipid imbalance among men. Overall, these results indicate that of the three tested professional groups, the highest global cardiovascular risk is found among bluecollar workers.

One of the most important findings is that in contrast to leisure-time PA, higher work-related PA (PA-EE) demonstrated no inverse association with cardiovascular risk factors. PA-EE demonstrates no association with BMI, WHR, lipid fractions or glucose in the whole studied population. This may indicate that PA related to work or occupational activity has no positive impact on basic cardiovascular risk profile, or that this impact is counterbalanced by other risk factors. Furthermore, it is possible that work-related PA may have a negative impact on some cardiovascular risk factors, iethat is, to increased BP. Among the whole group, PA-EE was connected with higher SBP. This correlation may be explained by the fact that blue-collar workers, those with highest PA-EE, presented increased BP values. Within the sample, the men with the greatest EE tended to present not only the lowest education level (blue-collar workers) but interestingly, also the lowest Hcy concentration. Some literature data indicate that PA may decrease Hcy level. ${ }^{40}$ Therefore, among the tested older subjects, the overall worse cardiometabolic risk profile displayed by blue-collar workers is counterbalanced by higher PA-EE, as compared with white-collar workers and unemployed subjects. However, PA-HRB appears to have a closer relationship with cardiometabolic risk profile than PA-EE, which may be related to the relationship between PA-EE profile and socioeconomic status.

Both moderate and hard PA-HRBs were found to exert beneficial impact on body mass indices and improve metabolic markers such as TG (both PA-HRB I and II), HDL-C (PA-HRB II) glucose (both PA-HRB I and II) or UA (PA-HRB I). All these correlations are consistent with previous findings. ${ }^{4-46}$

When analysed according to sex, these observations were found to apply particularly closely to the group of women. In the case of men, PA-HRB may decrease body mass indices (BMI, WHR) and improve the concentrations of lipids like TG or HDL-C; although, in case of lipids, this relationship was only observed for PA-HRB II. It should be emphasised that the Stanford Usual Activity questionnaire assesses different dimensions of non-work-related 
PA. PA-HRB I and PA-HRB II were not found to be interrelated; however, each was associated with PA-EE.

To the best of our knowledge, this is the first work to compare cardiovascular risk factors and different dimensions of PA in three groups of seniors according to type of the employment status. Its key strength is its sex and age-matching of subjects. However, the present cross-sectional study has some limitations. PA assessment tools (Seven Day Recall PA Questionnaire and the Stanford usual Activity Questionnaire) are self-reported subjective methods and the estimation of PA levels may be incorrect. Finally, our findings apply to balanced groups of Central-European pre-elderly people according to employment status and these values may be different in other populations and cultures.

\section{Practical implications}

All presented data suggest that promoting everyday life and leisure time PA behaviour is crucial for preventing cardiometabolic risk in pre-elderly subjects, even in bluecollar workers with high work-related EE. Whether that beneficial effect results only from PA per se or also from social and health awareness behaviours requires further prospective studies.

Contributors BKS: recruitment of subjects, medical history, physical examination, anthropometric examination, database preparation, basic statistical calculations, manuscript writing; JK: conducting questionnaire surveys on physical activity, performing statistical calculations on cardiovascular risk factors and physical activity, substantive supervision, manuscript drafting and revising; KK and CW: laboratory tests conduction, substantive supervision, manuscript revising; TK: preparing of the study design, performing statistical calculations, substantive supervision, manuscript drafting and revising

Funding The authors were supported by the Central Institute For Labour Protection: National Research Institute project (KBNSR/N///427/2014) and grants 503/6-077-01/503-61-002 and 502-03/6-077-01/502-64-110 from the Medical University of Lodz.

Competing interests None declared.

Patient consent for publication Parental/guardian consent obtained.

Ethics approval The study was approved by the Ethics Committee of the Medical University of Łódź (No RNN/648/14/KB). All subjects signed an informed consent form prior to participation in the study.

Provenance and peer review Not commissioned; externally peer reviewed.

Data availability statement № additional data available.

Open access This is an open access article distributed in accordance with the Creative Commons Attribution Non Commercial (CC BY-NC 4.0) license, which permits others to distribute, remix, adapt, build upon this work non-commercially, and license their derivative works on different terms, provided the original work is properly cited, appropriate credit is given, any changes made indicated, and the use is non-commercial. See: http://creativecommons.org/licenses/by-nc/4.0/.

\section{REFERENCES}

1. Prescott E, Hippe M, Schnohr P, et al. Smoking and risk of myocardial infarction in women and men: longitudinal population study. BMJ 1998;316:1043-7.

2. Kawachi J, Colditz GA, Stampfer MJ, et al. Smoking cessation in relation to total mortality rates in women. Ann Intern Med 1993;119:992-1000.

3. Laasko M. Insulin resistance and coronary heart disease. Curr Opin Lipidol 1996;7:217-26.

4. Tadic M, Cuspidi C, Pencic B, et al. The interaction between blood pressure variability, obesity, and felt ventricular mechanics: findings from the hypertensive population. J Hypertens 2016;34:772-80.
5. Meschia JF, Bushnell C, Boden-Albala B, et al. Guidelines for the primary prevention of stroke: a statement for healthcare professionals from the American heart Association/American stroke association. Stroke 2014:45:3754-832.

6. Wilking SVBet al. Determinants of isolated systolic hypertension. JAMA 1988;260.

7. Cordero Aet al. Comparison of long term mortality of cardiac diseases in patient with versus without diabetes mellitus. Am J Cardiol 2016;9149:30015-7.

8. Budoff MJ, Raggi P, Belle GA, et al. Noninvasive cardiovascular risk Assesment of the asymptomatic diabetic patient: the imaging Council of the American College of cardiology. JACC CadiovasC Imaging 2016;9:176-92.

9. Assmann G, Schulte H. Relation of high-density lipoprotein cholesterol and triglycerides to incidence of atherosclerotic coronary artery disease (the PROCAM experience). Am J Cardiol 1992;70:733-7.

10. Ganguly P, Alam SF. Role of homocysteine in the development of cardiovascular disease. Nutr J 2015;14:6

11. Robinson CF, Walker JT, Sweeney MH, et al. Overview of the National occupational mortality surveillance (NOMS) system: leukemia and acute myocardial infarction risk by industry and occupation in 30 us states 1985-1999, 2003-2004, and 2007. Am J Ind Med 2015;58:123-37.

12. Virkkunen $\mathrm{H}$, Härmä $\mathrm{M}$, Kauppinen $\mathrm{T}$. The triad of shift work, occupational noise, and physical workload and risk of coronary heart disease. Occup Environ Med 2006;63:378-86.

13. Krause N, Brand RJ, Wong CC, et al. Work time and 11-year progression of carotid atherosclerosis in middle-aged Finnish men. Prev Chronic Dis 2009;6.

14. Roupe S, Svanborg A. Previous job and health at the age of 70 Scand J Soc Med 1981;9:25-31.

15. Joseph NT, Muldoon MF, Manuck SB, et al. The role of occupational status in the association between job strain and ambulatory blood pressure during working and Nonworking days. Psychosom Med 2016;78:940-9.

16. Opit LJ, Oliver RG, Salzberg M. Occupation and blood pressure. Med J Aust 1984;140:760-4.

17. Babu GR, Jotheeswaran AT, Mahapatra T, et al. Is hypertension associated with job strain? A meta-analysis of observational studies. Occup Environ Med 2014;71:220-7.

18. Grippo AJ, Johnson AK, Stress JAK. Stress, depression and cardiovascular dysregulation: a review of neurobiological mechanisms and the integration of research from preclinical disease models. Stress 2009;12:1-21.

19. Pinsker EA, Hennrikus DJ, Hannan PJ, et al. Smoking patterns, quit behaviors, and smoking environment of workers in small manufacturing companies. Am J Ind Med 2015;58:996-1007.

20. Sorensen G, Pechacek T. Occupational and sex differences in smoking and smoking cessation. J Occup Med 1986;28:360-4.

21. Sorensen G, Gupta PC, Pednekar MS. Social disparities in tobacco use in Mumbai, India: the roles of occupation, education, and gender. Am J Public Health 2005;95:1003-8.

22. Helmert U. Cardiovascular risk factors and occupation: results of the health survey of the German cardiovascular prevention study. Soz Praventivmed 1996;41:165-77.

23. Perk Jet al. European guidelines on cardiovascular disease prevention in clinical practice (version 2012). Eur Heart $J$ 2012;33.13:1635-701.

24. Piepoli MFet al. ESC Scientific Document Group. European Guidelines on cardiovascular disease prevention in clinical practice: The Sixth Joint Task Force of the European Society of Cardiology and Other Societies on Cardiovascular Disease Prevention in Clinical Practice (constituted by representatives of 10 societies and by invited experts)Developed with the special contribution of the European Association for Cardiovascular Prevention \& Rehabilitation (EACPR). Eur Heart J 2016;37:2315-81.

25. Arem $\mathrm{H}$, Moore SC, Patel $\mathrm{A}$, et al. Leisure time physical activity and mortality: a detailed pooled analysis of the dose-response relationship. JAMA Int Med 2015;175:959-67.

26. Moore SC, Patel AV, Matthews CE, et al. Leisure time physical activity of moderate to vigorous intensity and mortality: a large pooled cohort analysis. PLoS Med 2012;9:e1001335.

27. Li J, Loerbroks A, Angerer P. Physical activity and risk of cardiovascular disease: what does the new epidemiological evidence show? Curr Opin Cardiol 2013;28:575-83.

28. Van Dam ROBM, Schuit AJ, Feskens EJM, et al. Physical activity and glucose tolerance in elderly men: the Zutphen elderly study. Medicine \& Science in Sports \& Exercise 2002;34:1132-6.

29. Gans KM, Salkeld J, Risica PM, et al. Occupation is related to weight and lifestyle factors among employees at Worksites involved 
in a weight gain prevention study. Journal of Occupational and Environmental Medicine 2015;57:e114-20.

30. Lim MS, Park B, Kong IG, et al. Leisure sedentary time is differentially associated with hypertension, diabetes mellitus, and hyperlipidemia depending on occupation. BMC Public Health 2017;17:278.

31. Kostka J, Kostka T, Borowiak E. Physical activity in older adults in relation to place of residence and coexistent chronic diseases. $J$ Phys Act Health 2017;14:20-8.

32. Sołtysik BK, Kroc Łukasz, Pigłowska M, et al. An evaluation of the work and life conditions and the quality of life in 60 to 65 year-old White-Collar employees, manual workers, and unemployed controls. Journal of Occupational and Environmental Medicine 2017;59:461-6.

33. White WB, Berson AS, Robbins C, et al. National standard for measurement of resting and ambulatory blood pressures with automated sphygmomanometers. Hypertension 1993;21:504-9.

34. van der Heijden AAWA, van't Riet E, Bot SDM, et al. Risk of a recurrent cardiovascular event in individuals with type 2 diabetes or intermediate hyperglycemia: the Hoorn study. Diabetes Care 2013;36:3498-502.

35. Alberti KGMM, Zimmet P, Shaw J. Metabolic syndrome-a new world-wide definition. A consensus statement from the International diabetes Federation. Diabet Med 2006;23:469-80.

36. Blair SN, Haskell WL, Ho P, et al. Assessment of habitual physical activity by a SEVENDAY recall in a community survey and controlled experiments. Am J Epidemiol 1985;122:794-804.

37. Sallis JF, Haskell WL, Wood PD, et al. Physical activity assessment methodology in the FIVE-CITY PROJECT1. Am J Epidemiol $1985 ; 121: 91-106$
38. Pereira MA, FitzerGerald SJ, Gregg EW, et al. A collection of physical activity questionnaires for health-related research. Med Sci Sports Exerc 1997;29(6 Suppl):S104-S106.

39. Bonnefoy M, Normand S, Pachiaudi C, et al. Simultaneous validation of ten physical activity questionnaires in older men: a doubly labeled water study. J Am Geriatr Soc 2001;49:28-35.

40. King GA, Fitzhugh EC, Bassett Jr DR, et al. Relationship of leisuretime physical activity and occupational activity to the prevalence of obesity. Int J Obes 2001;25:606-12.

41. Koskinen H-L, Kauppinen T, Tenkanen L. Dual role of physical workload and occupational noise in the association of the metabolic syndrome with risk of coronary heart disease: findings from the Helsinki heart study. Occup Environ Med 2011;68:666-73.

42. Korshøj M, Clays E, Lidegaard M, et al. Is aerobic workload positively related to ambulatory blood pressure? A cross-sectional field study among cleaners. Eur J Appl Physiol 2016;116:145-52.

43. Zhang J, Zhang Y, Deng W, et al. Elevated serum uric acid is associated with angiotensinogen in obese patients with untreated hypertension. J Clin Hypertens 2014;16:569-74.

44. Chin S-H, Kahathuduwa CN, Binks M. Physical activity and obesity: what we know and what we need to know*. Obesity Reviews 2016;17:1226-44.

45. Montesi L, Moscatiello S, Malavolti M, et al. Physical activity for the prevention and treatment of metabolic disorders. Intern Emerg Med 2013;8:655-66.

46. Chen J-H, Wen CP, Wu SB, et al. Attenuating the mortality risk of high serum uric acid: the role of physical activity underused. Ann Rheum Dis 2015;74:2034-42. 